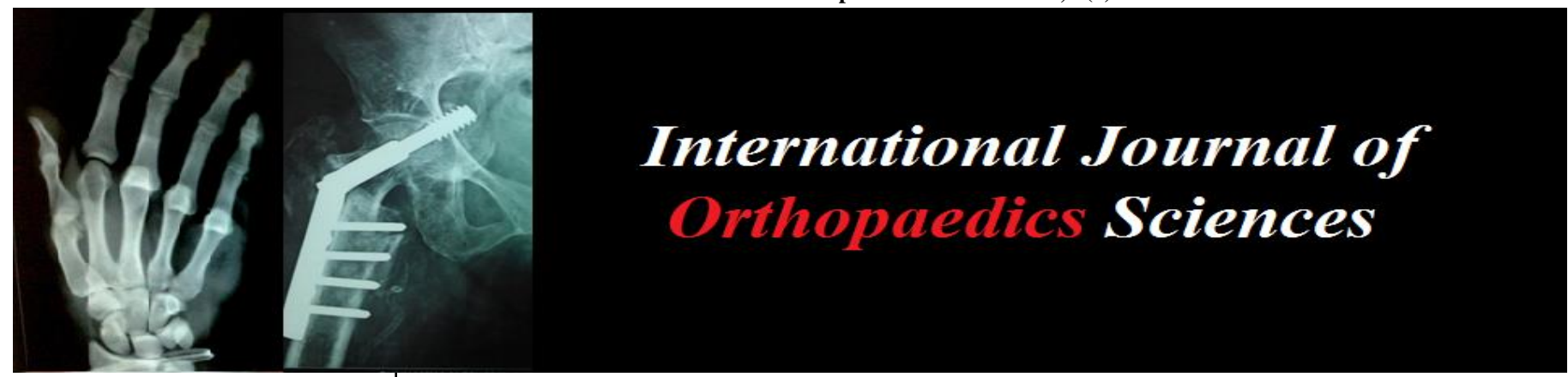

E-ISSN: 2395-1958

P-ISSN: 2706-6630

IJOS 2020; 6(1): 347-351

(C) 2020 IJOS

www.orthopaper.com

Received: 01-11-2019

Accepted: 05-12-2019

\section{Dr. Sanjay Deo}

Professor and HOD, Department

of Orthopaedics, Dr. D Y Patil

Medical College, Pune,

Maharashtra, India

Shubhanshu Gupta

Junior Resident, Department of Orthopaedics, Dr. D Y Patil

Medical College, Pune,

Maharashtra, India

Dr. Swaroop Solunke

Assistant Professor, Department of Orthopaedics, Dr. D Y Patil

Medical College, Pune,

Maharashtra, India

\section{Dr. Amol Patil}

Junior Resident,

Department of Orthopaedics,

Dr. D Y Patil Medical College,

Pune, Maharashtra, India

\section{Dr. Avinash Kuma}

Junior Resident, Department of

Orthopaedics, Dr. D Y Patil

Medical College, Pune,

Maharashtra, India

\section{Determination of bone mass and hip axis length of contralateral side in patients with hip fracture}

\author{
Dr. Sanjay Deo, Shubhanshu Gupta, Dr. Swaroop Solunke, Dr. Amol Patil \\ and Dr. Avinash Kumar
}

DOI: https://doi.org/10.22271/ortho.2020.v6.i1g.1887

Abstract

Introduction: The currently accepted definition of osteoporosis as follows: A progressive systemic skeletal disease characterized by a low bone mass and microarchitectural deterioration of bone tissue leading to an increase in bone fragility and a susceptibility to fractures. BMD compared with age and sex matched controls (Z-scores) or with young adult sex matched controls (T-scores). Hip axis length (HAL) is the dimension along the femoral neck axis from the base of greater trochanter through the femoral head and the acetabulum to the inner pelvic brim, and it is one of the strongest predictors of fracture risk.

Material and method: Twenty five patients with hip fracture of which 14 patients had intertrochantric fracture and 11 had neck femur fracture were included in our study. Bone mineral density of contralateral hip at neck, trochanteric region, Ward's triangle, upper shaft and total BMD were assessed. Hip axis length was calculated automatically by the machine. All the parameters were analyzed and compared for statistical reporting of results.

Summary and Conclusion: We conclude that the patients with hip fractures have significantly lower bone mass in proximal femur, femoral neck region and intertrochanteric region. The mean hip axis length of patients with femoral neck fractures $(10.25 \pm 0.73 \mathrm{~cm})$ is more than patients with intertrochanteric fractures $(9.76 \pm 0.80 \mathrm{~cm})$.

Keywords: Osteoporosis, intertrochantric fracture, neck femur fracture, bone mineral density, hip axis length, Dexa Scan

\section{Introduction}

The currently accepted definition of osteoporosis as follows: A progressive systemic skeletal disease characterized by a low bone mass and microarchitectural deterioration of bone tissue leading to an increase in bone fragility and a susceptibility to fractures ${ }^{[1]}$. Because clinically, osteoporosis is usually recognized by occurrence of low trauma fractures, thus any meaningful definition of osteoporosis must include fractures.

In 1994, the World Health Organization (WHO) defined the categories of osteoporosis in white Caucasian females using dual energy x-ray absorptiometry (DXA) measurements ${ }^{[2]}$.

BMD expressed as a percentage of the normal age-matched mean and the young adult mean. BMD compared with age and sex matched controls (Z-scores) or with young adult sex matched controls (T-scores). These values are calculated using normative data from a reference population. These values may be expressed as percentiles or standard deviation scores (SD). The Z- score is a measure of the difference between the patient's BMD and the mean BMD of age and sex matched peers. The T-score is a measure of the difference between the patient's BMD and the mean BMD of young normals. This information helps to provide an estimate of future fracture risk ${ }^{[2]}$.

The hip measurement sites include the femoral neck, Wards triangle (trabecular bone), greater trochanteric, and total hip regions. Hip axis length (HAL) is the dimension along the femoral neck axis from the base of greater trochanter through the femoral head and the acetabulum to the inner pelvic brim, and it is one of the strongest predictors of fracture risk ${ }^{[3]}$.
Corresponding Author: Shubhanshu Gupta Junior Resident, Department of Orthopaedics, Dr. D Y Patil Medical College, Pune,

Maharashtra, India 


\section{Aims and objectives}

To determine bone mineral density and hip axis length of the opposite hip (uninjured side) and to correlate with incidence of cervical and trochanteric hip fractures.

\section{Material and Methods}

The subjects included cases of admitted in DR D Y PATIL Medical College and Hospital, Pune with hip fractures from May 2017 to March 2019, those who were willing to participate in the study and were more than fifty years in age. Patients having illness causing prolonged stay in bed of 6 or more weeks prior to fracture and with secondaries or metastasis, rheumatoid arthritis, multiple myeloma, tubercular spine or with bilateral hip fracture were excluded from the study. The radiographs of pelvis with both hips AP view and lateral view of the injured side were done and classified as intertrochanteric fractures or fracture neck of femur.

Pre - anaesthetic check up of patients was done and patients were taken up for surgery. Closed reduction and internal fixation with a dynamic hip screw was done for intertrochanteric fractures, using C-Arm image intensifier. In patients with fracture neck of femur, either bipolar cemented hemiarthroplasty was done or closed reduction and internal fixation with cancellous cannulated screws was performed. A dual energy X-ray absorptiometry scan of the opposite hip of the patient was done either pre-operatively or within a week after surgery. Bone mineral density at neck, trochanteric region, Ward's triangle, upper shaft and total BMD were assessed. T-score and Z-score (young adult and age matched score) were calculated using normative data from reference population. Hip axis length was calculated automatically by the machine.

Patients were mobilized post operatively according to type of fixation and general health of the patient. At follow up patients were assessed at 6 weeks, 12 weeks and 6 months for fracture healing, position of implant, mobility of patient and any other fractures. All the parameters were analyzed and compared for statistical reporting of results.

\section{Observation and Results Age Incidence}

Table 1: The age distribution of patients with hip fractures was as follows

\begin{tabular}{|c|c|c|c|}
\hline \multirow{2}{*}{ Age (In Years) } & \multicolumn{3}{|c|}{ No. of Patients } \\
\cline { 2 - 4 } & Total & \# NOF & \# I/T \\
\hline $51-60$ & 3 & 2 & 1 \\
\hline $61-70$ & 11 & 4 & 7 \\
\hline $71-80$ & 7 & 3 & 4 \\
\hline $81-90$ & 4 & 2 & 2 \\
\hline
\end{tabular}

The mean age observed for hip fractures was 70.20 years with a standard deviation of 9.02 years, for femoral neck fractures it was 69.63 years with a standard deviation of 9.24 years and for intertrochanteric fractures it was70.64 years with a standard deviation of 9.22 years.

Sex distribution: Regarding the sex distribution of hip fractures the incidence among females (14) was about 1.27 times higher than males (11).

Type of fracture: Intertrochanteric fractures (14) were observed 1.27 times more often as compared to femoral neck fractures (11).

Height of patients: Mean height of the patients was $162 \pm$ 11.73 centimetres.

Weight of patients: Mean weight of patients was $60.44 \pm$ $8.26 \mathrm{Kg}$.

\section{BMD and t-score}

Total proximal femoral BMD and t-score

The mean total proximal femoral Bone Mineral Density was 0.693 , ranging from 0.534 to 0.832 with a standard deviation of 0.0769 and mean T-score was -3.07 , ranging from -4.2 to -1.8 with a standard deviation of 0.644 .

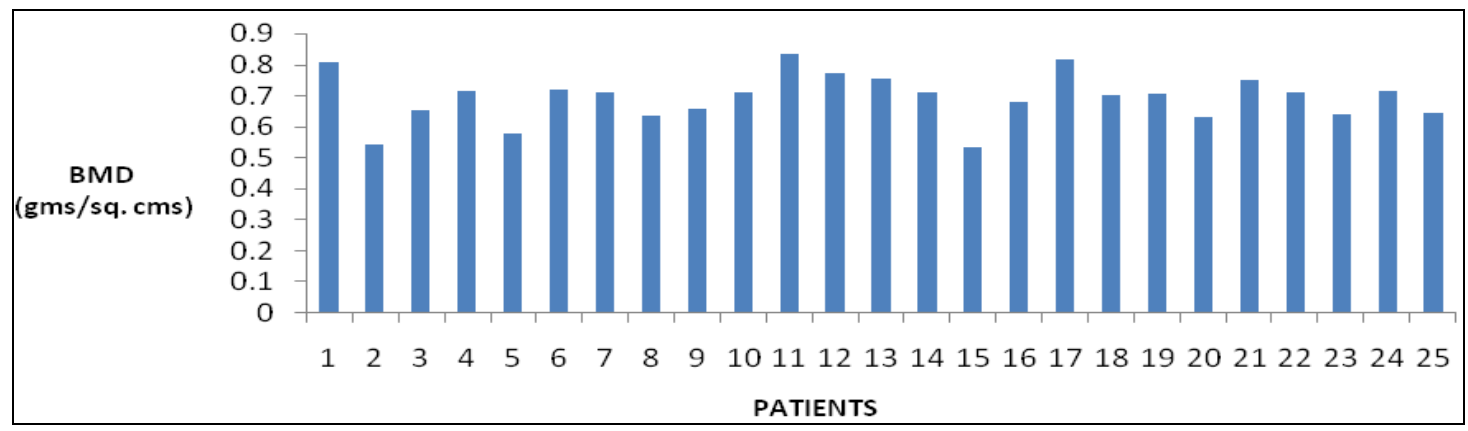

Fig 1: Total BMD in patients with HIP fracture

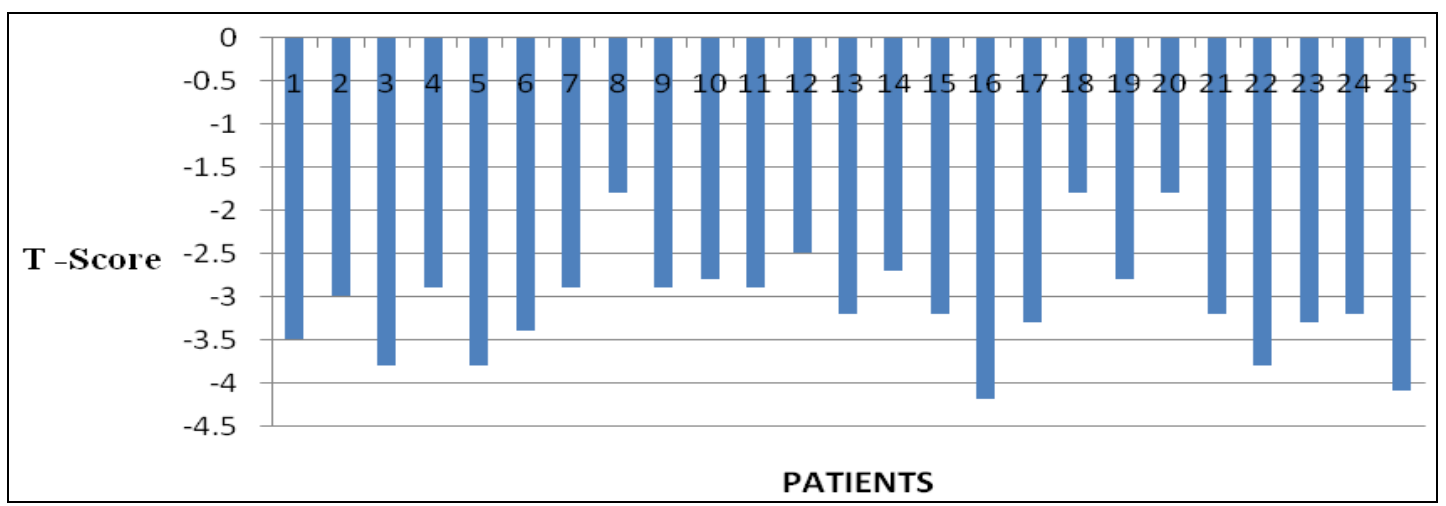

Fig 2: Total T Score in patients with HIP fracture $\sim 348 \sim$ 
In patients with femoral neck fractures the mean BMD was 0.672 ranging from 0.543 to 0.809 with a standard deviation of 0.074 and in patients with intertrochanteric fractures mean BMD was 0.709 ranging from 0.534 to 0.832 with a standard deviation of 0.077 .

In patients with femoral neck fracture the mean T- score was 3.15 , ranging from -4.1 to -1.8 with a standard deviation of 0.64 and in patients with intertrochanteric fractures mean $\mathrm{T}$ score was -3.0 , ranging from -4.2 to -1.8 with a standard deviation of 0.66

\section{BMD and T-score at femoral neck}

The mean Bone Mineral Density at femoral neck in patients with hip fractures was 0.668 , ranging from 0.519 to 0.773 . With a standard deviation of 0.069 and mean T-score was 3.02 , ranging from -3.9 to -1.6 with a standard deviation of 0.63 .

In patients with femoral neck fractures the mean BMD at femoral neck was 0.642 ranging from 0.519 to 0.765 with a standard deviation of 0.069 and in patients with intertrochanteric fractures mean femoral neck BMD was 0.689 ranging from 0.558 to 0.774 with a SD of 0.064 .

In patients with femoral neck fractures the mean $\mathrm{T}$ - score at femoral neck was -3.145 , ranging from -3.9 to -1.9 with a standard deviation of 0.636. and in patients with intertrochanteric fractures mean $\mathrm{T}$ score at femoral neck was -2.92 , ranging from -3.9 to -1.6 with a standard deviation of 0.639 .

\section{BMD and T-Score at intertrochanteric region}

Mean BMD at intertrochanteric region in patients with hip fractures was 0.58 ranging from 0.418 to 0.701 with a standard deviation of 0.073 and mean T-score was-2.69, ranging from -4.4 to-1.4 with a standard deviation of 0.797

Mean BMD at intertrochanteric region in patients with femoral neck fracture was 0.552 ranging from 0.418 to 0.659 with a standard deviation of 0.078 and in patients with fracture intertrochanteric it was 0.602 ranging from 0.438 to 0.658 with a standard deviation of 0.0643 .

In patients with femoral neck fracture the mean $\mathrm{T}$ - score at intertrochanteric region was -2.73 , ranging from -4.3 to -0.9 with a standard deviation of 0.84 . and in patients with intertrochanteric fractures mean $\mathrm{T}$ score was -2.66 , ranging from -4.4 to -1.4 with a standard deviation of 0.787 . In patients with femoral neck fractures the mean T-score at neck region was $-3.491 \pm 1.46$, and at intertrochanteric region was $-2.74 \pm 0.84$. The distribution of T-scores at femoral neck region and intertrochanteric region in these patients was as follows:

In patients with intertrochanteric fractures the mean $\mathrm{T}$-score at neck region was $-2.92 \pm 0.63$, and at intertrochanteric region was $-2.66 \pm 0.79$.

\section{Z-Score}

The mean Z-score in patients with hip fractures was -1.37 , with a standard deviation of 0.72 .

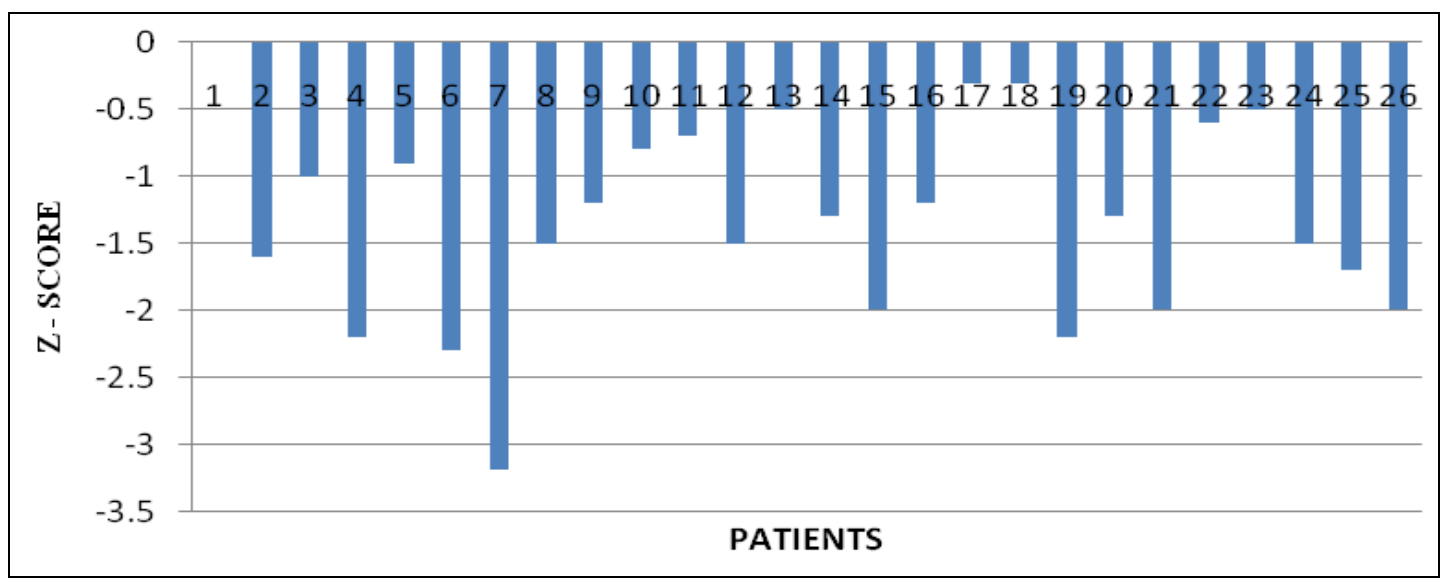

Fig 3: Z-score in Patients with HIP fracture

Hip axis length (HAL)

The mean hip axis length of patients with hip fractures was
$9.9 \mathrm{cms}$, ranging from 8.97 to 11.62 with standard deviation of $0.796 \mathrm{~cm}$.

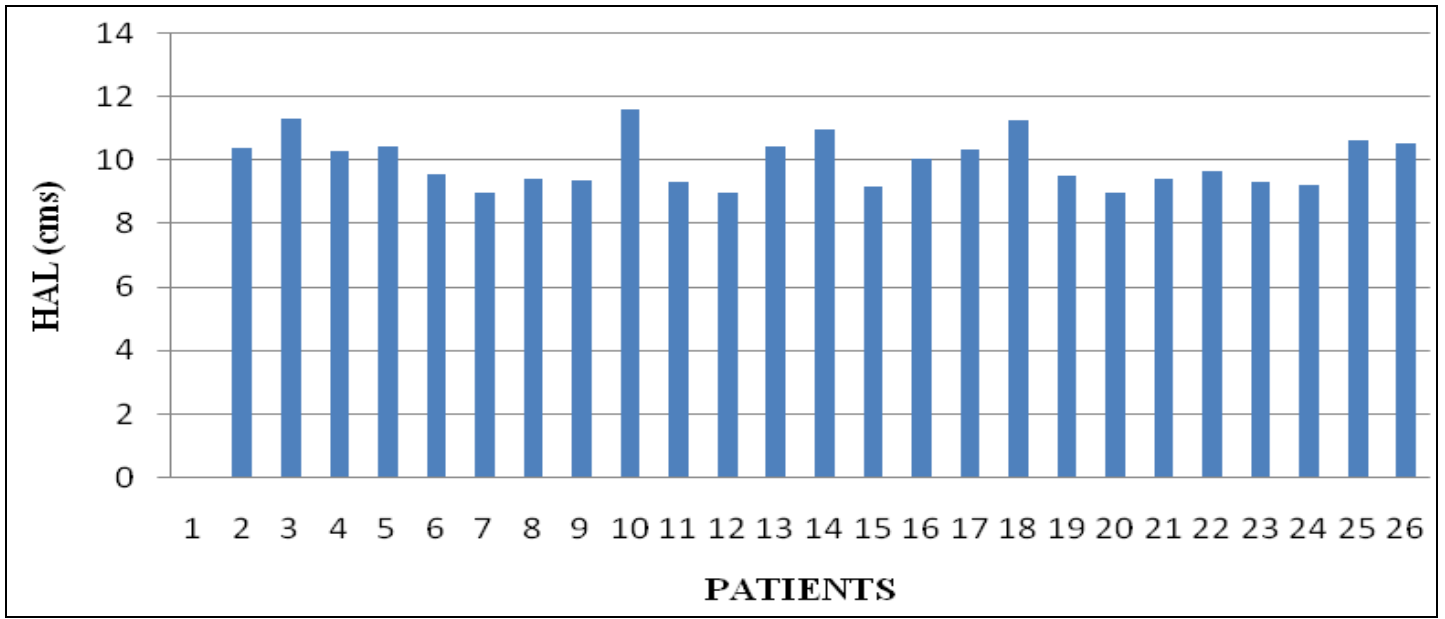

Fig 4: Hal in patients with HIP fracture 
The mean hip axis length of patients with femoral neck fractures was $10.25 \mathrm{~cm}$, ranging from 9 to 11.31 with standard deviation of $0.73 \mathrm{~cm}$. and in patients with intertrochanteric fractures it was $9.76 \mathrm{~cm}$, ranging from 8.97 to 11.62 with a standard deviation of $0.802 \mathrm{~cm}$.

\section{Distribution of HAL in male and female patients}

In male patients the mean HAL was $10.71 \mathrm{~cm}$ with a standard deviation of $0.51 \mathrm{~cm}$ and in female patients mean HAL was $9.39 \mathrm{~cm}$ with a standard deviation of $0.37 \mathrm{~cm}$.

\section{Type of treatment}

All the fourteen patients with intertrochantric fracture were treated with dynamic hip screw.

Out of eleven patients with femoral neck fractures, eight had bipolar hemiarthroplasty and three patients had closed reduction and internal fixation with cancellous cannulated screws.

\section{Fracture healing}

Fracture healing was assessed in patients with intertrochanteric fractures, and femoral neck fractures treated with cancellous cannulated screws upto six months from time of fracture. All the fourteen patients treated with dynamic hip screw showed union at the end of 6 months. Out of three femoral neck fracture patients managed with multiple pinning, one patient's fracture united and two had non union.

\section{Position of implant}

Out of fourteen patients with intertrochanteric fractures treated with dynamic hip screw, eight had collapse at fracture site at six weeks follow up. Out of these eight three had further collapse at twelve weeks and one patient had lag screw cut through at six months. All the eight patients treated with bipolar hemiarthroplasty showed no change in position of implant upto six months after surgery. Out of the three patients with femoral neck fracture treated with multiple pinning, one had collapse and back out of screws with fracture healing on follow up at six and twelve weeks and other two had cut through of screws at twelve weeks.

\section{Mobility of patient}

Out of twenty five patients, all were mobile with walker at six weeks and at twelve weeks, eleven patients were still mobile with walker, while fourteen were walking with stick. At six months, four patients were walking with walker, nine with a stick and twelve patients were walking independently.

\section{Complications}

Out of fourteen patients with intertrochanteric fractures, one patient had cut through of cervical lag screw, but the fracture had united and metal work was removed eleven months after fixation. None of the eight patients managed with bipolar hemiarthroplasty had any complications. Out of three patients with femoral neck fracture treated with multiple pinning, one had impingement of screws eleven months after surgery, his fracture had united and he was moving independently, he was advised removal of implant. Other two patient had collapse and cut through of screws with non union of fracture when last seen at 12 weeks after surgery and were advised arthroplasty.

\section{Summary and Conclusion}

This prospective study was conducted at Dr. D Y Patil Medical College and Hospital, Pune to determine the bone mineral density and hip axis length of the opposite side (uninjured side) in patients with hip fractures. Twenty five cases of hip fractures (eleven femoral neck fractures and fourteen intertrochanteric fractures) were finally evaluated.

The mean age observed for hip fractures was $70.20 \pm 9.02$ years. For femoral neck fractures mean age was $69.63 \pm 9.24$ years and for intertrochanteric fractures mean age was 70.64 \pm 9.22 years. The mean age observed for hip fractures and intertrochantric fractures is comparable with other studies but we have observed neck of femur fracture in relatively younger population ${ }^{[3,4,5]}$

Intertrochanteric fractures were observed 1.27 times more often as compared to femoral neck fractures and the incidence of hip fractures among females was about 1.27 times higher than males. These findings were not in accordance with observations of other workers ${ }^{[6,7]}$.

It was observed that left side (non dominant side) was injured more commonly. The height and weight distribution of patients was similar to as reported in literature ${ }^{[4,5]}$ All the patients suffered fractures as a result of trivial fall.

The mean total proximal femoral Bone Mineral Density observed in patients with hip fractures was $0.693 \pm 0.0769$ $\mathrm{gm} / \mathrm{sq} . \mathrm{cm}$ and mean total proximal femoral T-score in patients with hip fractures was $-3.07 \pm 0.644$. These values are significantly lower in comparison to values of control populations reported by other workers, suggesting that the patients with hip fractures have significantly lower bone mass in proximal femur, this finding supports the data in literature [7-9]

We observed that in patients with femoral neck fractures the mean total proximal femoral BMD was $0.672 \pm 0.074$ and in patients with intertrochanteric fractures the mean total proximal femoral BMD was $0.709 \pm 0.077$. Mean total proximal femoral $\mathrm{T}$-score in patients with femoral neck fractures was $-3.15 \pm 0.64$, and in patients with intertrochanteric fractures was $-3.0 \pm 0.66$. So we observed that bone mass is lower in patients with femoral neck fractures but our findings are not in accordance with literature $[5,7,10]$

The mean Bone Mineral Density at femoral neck in patients with hip fractures was $0.668 \pm 0.069$ and mean T-score was $3.02 \pm 0.63$ and our finding that patients with hip fractures have significantly lower BMD and T-scores at femoral neck region as compared to controls taken from other studies is in accordance with observations of other workers $[3,4,9,11]$

In patients with femoral neck fractures the mean BMD at femoral neck was $0.642 \pm 0.069$ and mean T-score was -2.89 \pm 0.68 and in patients with intertrochanteric fractures mean femoral neck BMD was $0.689 \pm 0.064$ and the mean T score was $-2.92 \pm 0.639$. So, in our present study the bone mass at femoral neck region is less in patients with femoral neck fractures as compared to patients with intertrochanteric fractures which is contrary to observation of other workers ${ }^{[6]}$. The mean Bone Mineral Density at intertrochanteric region in patients with hip fractures was $0.58 \pm 0.073$ and our finding that patients with hip fractures have significantly lower BMD at intertrochanteric region as compared to controls taken from other studies is in accordance with observations of other workers ${ }^{[9]}$.

Mean BMD at intertrochanteric region in patients with femoral neck fractures was $0.552 \pm 0.078$ and the mean $\mathrm{T}$ score was $-2.73 \pm 0.84$ and in patients with intertrochanteric fractures the mean BMD at intertrochanteric region was 0.602 \pm 0.643 and mean $\mathrm{T}$ score was $-2.66 \pm 0.787$. These findings 
suggest that bone mass at intertrochanteric region is less in patients with femoral neck fractures as compared to patients with intertrochanteric fractures which is contrary to observation of other workers ${ }^{[6]}$.

In patients with femoral neck fractures the mean $\mathrm{T}$-score at neck region was $-3.145 \pm 0.636$, and at intertrochanteric region was $-2.92 \pm 0.639$. In patients with intertrochanteric fractures the mean $\mathrm{T}$-score at neck region was $-2.92 \pm 0.63$, and at intertrochanteric region was $-2.66 \pm 0.79$. This observation implies that $\mathrm{T}$ - scores at both regions are lower in patients with femoral neck fractures and further, irrespective of the type of fracture the T- scores are lower at femoral neck region as compared to intertrochanteric region.

The mean hip axis length of patients with hip fractures in our study was $9.9 \pm 0.796 \mathrm{~cm}$. Although other studies show that hip axis length is more in patients with hip fractures, in absence of a control population if we compare our findings with those of others, our patients still have a lesser HAL which may be attributable to small sample size and smaller body frame of Indian population ${ }^{[4,5,8,12]}$.

The mean hip axis length of patients with femoral neck fractures was $10.25 \pm 0.73 \mathrm{~cm}$ and in patients with intertrochanteric fractures it was $9.76 \pm 0.80 \mathrm{~cm}$. This finding is in accordance with literature, that patients with femoral neck fractures have longer hip axis length as compared to patients with intertrochanteric fractures or in other words patients with longer HAL are more prone to femoral neck fractures.

In male patients the mean HAL was $10.65 \pm 0.49 \mathrm{~cm}$, and in female patients mean HAL was $9.36 \pm 0.37 \mathrm{~cm}$. This suggests that males have longer HAL than females which may be attributable to larger body frame of male population.

The union rate in surgically treated patients with intertrochanteric fractures was $100 \%$, and in literature also the non union rate in such patients is reported to be about $2 \%$. The non union rate in femoral neck fractures has been reported from $0 \%$ to over $30 \%$, but in our study only three patients of femoral neck fractures had internal fixation (rest had hemiarthroplasty), out of which two had non union and this number of patients is too small to be statistically significant.

Regarding the position of implant at follow up, out of fourteen patients with intertrochanteric fractures treated with dynamic hip screw, one patient had cervical lag screw cut through at six months. In literature the rate of loss of fixation has been reported from $4 \%$ to $20 \%$. So our observation of one lag screw cut-out falls within this range. The total BMD of this patient was $0.679 \mathrm{gm} / \mathrm{sq} . \mathrm{cm}$ and $\mathrm{T}$-score of -4.2 . Out of the eight patients treated with bipolar hemiarthroplasty we observed no change in position of implant upto six months after surgery. Out of the three patients with femoral neck fracture treated with multiple pinning, one had collapse at fracture site and back out of screws on follow up at six and twelve weeks. The other two had cut through of screws at twelve weeks, the total BMD of this patients were 0.554 grams $/ \mathrm{sq} . \mathrm{cm}$ and $0.638 \mathrm{gms} / \mathrm{sq} . \mathrm{cms}$ on the other hand the Tscore were -3.8 and-3.3 which is first and third lowest among the twenty five patients. So in this patient the loss of fixation may have occurred due to very poor bone quality.

Regarding the mobility of patients, at six months follow up twelve patients were walking independently, nine with a stick and only four patients were walking with walker, So about $50 \%$ patients reached the pre-injury ambulatory status at six months follow up.

We conclude that the patients with hip fractures have significantly lower bone mass in proximal femur, femoral neck region and intertrochanteric region. We have observed intertrochanteric fractures in patients with lower bone mass and at a older age as compared to patients with femoral neck fractures. The mean hip axis length of patients with hip fractures is $9.98 \pm 0.796 \mathrm{~cm}$. and the mean hip axis length of patients with femoral neck fractures $(10.25 \pm 0.73 \mathrm{~cm})$ is more than patients with intertrochanteric fractures $(9.76 \pm 0.80 \mathrm{~cm})$. For further studies, we suggest to have a reference population data pertaining to healthy age and sex matched ethnic Indian population. This could be of paramount importance for expected further epidemiological control of osteoporotic fractures.

\section{Reference}

1. Concensus Development Conference. Diagnosis, Prophylaxis and Treatment of Osteoporosis Am. J Med. 1993; 94:646-650.

2. World Health Organization WHO technical report series No. 843. Assesment of fracture risk and its application to screening for postmenopausal Osteoporosis, 1994.

3. Faulkner KG, Cummings SR, Black D, Palermo L, Gluer CC, Genant HK. Simple measurement of femoral geometry predicts hip fracture: The study of osteoporotic fractures. J Bone Miner Res. 1993; 8(10):1211-7.

4. Gnudi S, Ripamonti C, Gualtieri G, Malavolta N. Geometry of proximal femur in the prediction of hip fracture in osteoporotic women. Br J Radiol. 1999; 72(860):729-33.

5. Rosso R, Minisola S. Hip axis length in an Italian osteoporotic population. The British Journal of Radiology. 2000; 73:969-972.

6. Stewart A, Porter RW, Primrose WR, Walker LG, Reid DM. Cervical and trochanteric hip fractures: bone mass and other parameters. Clin Rheumatol. 1999; 18(3):2016.

7. Vaidya SV, Dholakia D, Yadav S. An age- and sexcontrolled matched pair analysis of $\mathrm{T}$ scores in ethnic Indians with hip fractures. J Orthop Surg. (Hong Kong). 2003; 11(1):22-7.

8. Alekel DL, Mortillaro E, Hussain EA, West B, Ahmed N, Peterson CT, Werner RK et al. Lifestyle and biologic contributors to proximal femur bone mineral density and hip axis length in two distinct ethnic groups of premenopausal women. Osteoporosis Int. 1999; 9(4):32738.

9. Cummings SR, Black DM, Nevitt MC, Browner W, Cauley J, Ensrud $\mathrm{K}$ et al. Bone density at various sites for prediction of hip fractures. The Study of Osteoporotic Fractures Research Group. Lancet. 1993; 341(8837):725.

10. Baudoin C, Fardellone P, Sebert JL. Effect of sex and age on the ratio of cervical to trochanteric hip fractures, A meta-analysis of 16 reports on 36,451 cases. Acta Orthop Scand. 1993; 64:647-653.

11. Mehta G, Taylor P, Petley G, Dennison E, Cooper C, Walker-Bone K. Bone mineral status in immigrant IndoAsian women. QJM. 2004; 97(2):95-9.

12. Ng CE, Sundram F, Mahbub UR, Hoon P. Bone mineral densities and hip axis lengths of normal Singapore women. Med J Malaysia. 1999; 54(2):180-4. 